\title{
Assessing the organizational characteristics influencing quality improvement implementation in Saudi hospitals
}

\begin{abstract}
Background: The healthcare system in Saudi Arabia has serious problems with quality and safety that can be reduced through systematic quality improvement (QI) activities. Despite the use of different quality improvement models to improve healthcare in Saudi hospitals during the last two decades, consistent improvements have not yet been achieved and the results are still far below expectations. This may reflect the presence of hidden organizational factors in the local contexts that hinder quality improvement efforts. The objective of this study is to assess the extent of quality improvement implementation in Saudi hospitals and to identify the organizational characteristics that make Saudi hospitals particularly challenging for quality improvement. Understanding these characteristics can inform efforts to improve them and may lead to more successful implementation.
\end{abstract}

Methods: The population under study is the individuals working as quality directors in MOH hospitals in all the provinces of Saudi Arabia. This population features members of the hospitals who have depth knowledge and experience in the research topic and can provide rich information about the factors impeding or underpinning quality improvement in Saudi hospitals. A mixed-method approach was conducted using two data collection tools: questionnaires and interviews. The quantitative phase (questionnaires) aimed to uncover the current level of quality improvement implementation in Saudi hospital as measured by seven critical dimensions adapted from the literature. The qualitative phase (interviews) aimed to understand the organizational characteristics that impede or underpin quality improvement in Saudi hospitals.

Results and discussion: The quality improvement implementation was found to be significantly poor across the seven dimensions with average score ranging between $(22.80 \pm 0.57)$ and $(2.11 \pm 0.69)$ on a 5 Likert scale. We also found that, the current level of QI implementation neither helped Saudi hospitals to improve 'customer satisfaction' nor to achieve measurable improvements in 'quality results' scoring significantly low at $(2.11 \pm 0.69)$ with $(\mathrm{p}$-value $=0.000)$ and $(2.47 \pm 0.57)$ with $(\mathrm{p}$-value $=0.000)$ respectively. Our study confirms the presence of a multitude of organizational barriers that impede QI in Saudi hospitals. These are related to: organizational culture, human resources management, processes and systems, and structure. These four were found to have the strongest impact on QI in Saudi hospitals.

Conclusion: It appears that the most important contributing factors to the successful implementation of quality improvement in Saudi hospitals are proper human resources utilization and effective quality management. Through careful planning, change management, proper utilization of human resources, supportive quality information systems, focus on processes and systems, structural support, and an organizational culture that is compatible with QI philosophy, Saudi hospitals will be more capable in achieving sustained measureable improvements in the quality and safety of patient care.

Keywords: quality improvement, quality management, Saudi Arabia
Volume 5 Issue 4 - 2018

\author{
Mohammad Shamsuddin Alaraki \\ Department of Healthcare Quality,Avenue Medical Center, King \\ Fahad Specialist Hospital, Saudi Arabia
}

Correspondence: Mohammad Shamsuddin Alaraki, Department of Healthcare Quality, Avenue Medical Center, King Fahad Specialist Hospital, Saudi Arabia, Email nilevision@hotmail.com

Received: June 03, 2018 | Published: July 09, 2018

\section{Background}

Quality improvement in healthcare has been studied intensively in the advanced countries of Europe and America; however, less research has come from developing countries and fewer studies have concerned Saudi Arabia. This country is different from other developing countries because its income is higher than that of many other such countries and the government is extremely willing to support performance measurement and continuous quality improvement in all the hospitals of the Saudi Kingdom. As stated by the Minister of Health in his foreword to the third edition of the standards of the Saudi Centre for Accreditation of Healthcare Institutions (CBAHI), 2015: "The healthcare industry in Saudi Arabia is experiencing an evolution associated mainly with one of the fastest growing rates of population in the world and a remarkable economic prosperity. This has been paralleled by a significant and steady improvement in the overall performance of the Saudi health sector secondary to the never-ending government support and the several quality programs and initiatives". Khalid AlFalih, 
This reflects a strong support and commitment of the Saudi government to quality improvement and a long term development strategy to improve the quality and safety of patient care in Saudi Arabia. ${ }^{1}$ Other drivers for improved care and services are the rising costs, increasing demand for healthcare services, a rise in medical errors, long waiting times, and the high expectations of the Saudi citizens of the healthcare system. ${ }^{2,3}$ However, the level of QI implementation and the extend of challenges faced by Saudi hospitals in applying QI strategies are still not clear. ${ }^{1}$ However, the poor results of quality initiatives in Saudi hospitals are well documented in the literature. A number of studies have been carried out to explain this variation from expectations. Among the most prominent studies were those conducted by Albejaidi ${ }^{4}$ and Al Malki et al. ${ }^{5}$ who maintained that Saudi hospitals were facing serious challenges in improving the quality of services provided to citizens despite the enormous fiscal expenditure and huge resources allocated by the Saudi government to the health sector? Other studies by Alahmadi \& Roland, ${ }^{6}$ Jannadi et al. ${ }^{7}$ Walston et al. ${ }^{8}$ showed that the Saudi healthcare system does not provide a well-developed quality culture where quality improvement strategies and other quality initiatives can flourish. Aljuaid, et al. ${ }^{3}$ in their recent study, clearly highlighted the need to improve the quality of healthcare delivery in Saudi hospitals, specifically in areas of patient safety, clinical effectiveness and patient-centeredness. It should be noted, however, that many of these previous studies were limited because they were not based on empirical evidence reflecting the internal organizational characteristics that facilitate or hinder QI implementation in Saudi hospitals. In addition, very few studies presented a comprehensive approach to measure quality improvement implementation. Hence, the picture of the QI application in Saudi hospitals and the complexity of the challenges they may be facing is not yet clear. Therefore, this study assesses the level of Quality Improvement implementation in Saudi hospitals and identifies the organizational characteristics that facilitate or hinder quality improvement.

\section{Methods}

The aim of this study was to evaluate the level of quality improvement implementation in Saudi hospitals and to identify the factors that facilitate or hinder sustained QI. The framework of this study consisted of two phases in which we used a mixedmethod approach for data collection. In the first phase, we used a questionnaire adapted from Quinn \& Kimberly ${ }^{9}$ to measure QI implementation in Saudi hospitals. In the second phase we used semistructured interviews to understand the organizational characteristics that impede or underpin quality improvement in Saudi hospitals.

\section{The questionnaire}

In the present study, the degree of QI implementation was defined as the extent to which the Saudi hospitals apply the QI concepts and practices in its management system. The level of QI implementation was measured using a questionnaire adapted from a survey developed by Quinn \& Kimberly'. The survey incorporates seven dimensions and 50 question items that address organization-wide QI efforts to improve the quality of care and services. These are: leadership, information and analysis, strategic quality planning, human resource utilization, quality management, quality results, and customer satisfaction. The following is a brief description of each.

Leadership: is measured by 10 practices: support to QI, driving QI efforts, allocating adequate resources, participation in QI activities, setting clear vision, managing change, acting on improvement suggestions, engagement of physician leaders, thorough understanding of QI, and confidence on success of QI efforts.

Information and analysis: is measured by 7 practices: involving employees in determining what data to collect, collecting data to measure performance, improving accuracy of the data, improving timelines of data collection, improving the use of data, using data in QI, and comparing data with other top performing hospitals.

Strategic quality planning: is measured by 6 practices: giving employees time to participate in QI activities, setting goals for QI aligned with the hospital strategic goals, disseminating goals to employees, involving employees in developing plans for QI, involving middle management and frontline employees in setting priorities for QI.

Human resources utilization: is measured by 8 practices: employees' education and training in QI, data management, and job skill and performance, employee rewarding and recognition, inter-departmental collaboration, empowerment of employees, supporting employees in take risks, and encouraging employees to make suggestions for improvement.

Quality management: is measured by 7 practices: regular checking of equipment and supplies, coordination of QI efforts, implementing effective policies and procedures, relationship with suppliers, building quality in new services, continuous improvement, and keeping records for QI measures.

Quality results: is measured by 5 practices: measurable improvements in healthcare, measurable improvements in clinical support departments, measurable improvements in non-clinical support services, measurable improvements in patient satisfaction results, and measurable improvements in cost reduction.

Customer satisfaction: is measured by 7 practices: assessing the current and future patient needs and expectations, handling patients' complaints, using feedback from patient to improve services, communicating patient satisfaction to hospital staff, assessing physician satisfaction, and assessing employee satisfaction.

\section{Pilot study of the questionnaire}

Five hospitals were selected for the pilot study of the questionnaire using convenient snowball sampling techniques and the researcher's personal experience and knowledge of the Saudi hospitals. The purpose of the pilot study was to refine the questions and check their applicability to the local context. The feedback was used in modifying the questionnaire to avoid any confusion or ambiguity. Based on the feedback the original survey of Quinn and Kimberly was decreased to 50 questions.

\section{Study population and data collection}

The quantitative phase of the study was restricted to $\mathrm{MOH}$ hospitals in all the provinces of Saudi Arabia. Military hospitals and National Guard hospitals were excluded because of difficulties in accessing these hospitals. Out of $220 \mathrm{MOH}$ hospitals involved in the study, a random sample of 60 hospitals was selected from different provinces representing approximately $27 \%$ of the $\mathrm{MOH}$ hospitals in Saudi Arabia. The sample was limited to quality directors in the selected hospitals because they have the professional knowledge and experience in quality improvement science and could provide accurate information 
about the current level of QI in their hospitals. Other professionals such as leadership and clinical staff were excluded because they have their own views to quality improvement. Besides most of them lack the technical knowledge about quality and their feedback would cause great bias and error in the current research. The survey was emailed to the directors of quality departments at each of the hospitals. A cover letter was attached to explain the nature and purpose of the research, and the directors were asked to complete the survey and to return it by email. After two reminders to non-respondents, a total of 47 completed questionnaires were received (response rate $78 \%$ ). Four responses were excluded due to incomplete answers for key data elements. The number of complete questionnaires used in the analysis was 43 (effective response rate $71.7 \%$ ).

\section{Semi-structured interviews}

The population of the qualitative phase of the study was also the individuals working as quality directors in $\mathrm{MOH}$ hospitals in Saudi Arabia. This population features members of the hospitals who have depth knowledge and experience in the research topic and can provide rich information about the challenges that have the potential to impede, disrupt or derail application of quality improvement in Saudi hospitals. A mixed purposeful sampling technique was used, namely: criterion and critical case. The sampling started with criterion sampling strategy where all cases that meet some criteria were selected. The criteria for selection included: relevance and depth of their experience in the field of research, knowledge about quality improvement methods and tools, and past experience in conducting quality improvement projects in the hospital. Criterion sampling was followed by critical case sampling. From those who met the criteria, cases that will produce critical information about the characteristics of Saudi hospitals that facilitate or hinder quality improvement were selected. The sample size was 12 quality directors representation most of the 13 regions of Saudi Arabia.

\section{Data analysis}

To analyse the data of the quantitative phase, counts and percentages $\mathrm{n}(\%)$ were used to summarize all categorical characteristic variables, while means plus/minus standard deviations (Mean $\pm \mathrm{SD}$ ) used for continuous variables. The 5-Likert scale has been used in the questionnaire with the following values: strongly disagree (1), disagree (2), neither (3), agree (4), and strongly agree (5). The mean and the standard deviation of the different questions were calculated. It was considered that the manager or the employee agree on a given statement when the mean is (3.5) or more. A mean below this value, indicate a negative response. Full Pearson's correlation coefficient was performed incorporating all the predictors from the seven domains, in order to examine the independent associations between each of these factors and the degree of QI implementation. Thematic Analysis (TA) approach was used to analyze the data of the qualitative phase.

The approach involved a six-step process:

i. Familiarisation with the data: this involved reading and rereading the data to become immersed and intimately familiar with its content.

ii. Coding: generating succinct labels (codes) that identify important features of the data that might be relevant to answering the research question.

iii. Searching for themes: examining the codes and collated data to identify potential themes. iv. Reviewing themes: checking the candidate themes against the dataset to determine that they tell a convincing story of the data.

v. Defining and naming themes: developing a detailed analysis of each theme, working out the scope and focus of each theme.

vi. Writing up: weaving together the analytic narrative and data extracts, and contextualising the analysis in relation to existing literature.

Data from interviews was coded and categorized in themes to facilitate analysis. To minimize the chance of errors from coding and increase the reliability of the data the coding was carried by three coders working independently on the same data. The coding consisted of a two-step process beginning with basic coding in order to distinguish overall themes, followed by a more in depth, interpretive code in which more specific trends and patterns could be interpreted. To ensure code is consistent, clear instructions and guidelines were given to coders that each code should apply to only one category and categories should be comprehensive. The process was done manually by highlighting different concepts with different colours.

\section{Results}

\section{Results of the questionnaire}

\section{Demographic characteristics of participants}

General characteristics of the participants are shown in Table 1. Out of 43 participants 34 (79.07\%) were males, and 9 (20.93\%) were females. Regarding the age $13(30.23 \%)$ participants were found to be 40 to 49 years old, while $30(69.77 \%)$ were above 50 years old. Those with bachelor degree were $13(30.23 \%)$ while participants with post graduate degrees were $30(69.77 \%)$. The majority were Physicians 29 (67.44\%), while administrators were only 14 (32.56\%). The work experience was more than 10 years for all the participants $43(100.00 \%)$.

Table I Demographic characteristics $(n=43)$

\begin{tabular}{ll}
\hline Characteristics & N (\%) \\
\hline Gender & $34(79.07 \%)$ \\
Males & $09(20.93 \%)$ \\
Females & \\
Age & $13(30.23 \%)$ \\
$40-49$ years & $30(69.77 \%)$ \\
$\geq 50$ years & \\
Education & $13(30.23 \%)$ \\
Bachelor & $30(69.77 \%)$ \\
Master/Doctorate & \\
Staff position & $29(67.44 \%)$ \\
Physician/dentist & $14(32.56 \%)$ \\
Administrator & \\
Experience & $43(100.00 \%)$ \\
More than 10 years &
\end{tabular}

\section{Impact of the demographic characteristics of participants on quality improvement implementation}

The t-test for two independent variables revealed no significant relationship between the demographic characteristics of respondents and the level of quality improvement implementation ( $p$-value $>0.05$ ). However, as shown in Table 2, we found that information and analysis was significantly associated with age group (p-value 0.010 ), education level (p-value 0.010), and staff position (p-value 0.005). 


\section{Degree of QI implementation in Saudi hospitals}

One Sample t-test was used to compare the statistical difference between the sample mean and a theoretical value of the mean in the population to determine whether the two means are significantly different. As shown in Table 3, the general level of QI implementation in Saudi hospitals was significantly low across the seven dimensions with p-value $<0.05$.

\section{Correlation between the dimensions of QI implementation}

As shown in Table 4, Pearson's correlation coefficient revealed the association between the dimensions of QI implementation.

\section{Result of the interviews}

Twelve individual interviews were carried out with quality experts in different Saudi hospitals, all of whom were seniors with average age of 48 years and more than 18 years experience. Each interview took an average of 55 minutes. Besides face-to-face, the interviews were carried out using modern communication technologies such as Skype, IMO, and LINE.

\section{Organizational factors impeding $\mathbf{Q}$ implementation}

The views of participants on organizational factors impeding quality improvement and sustainability were grouped into four main themes and 36 sub-themes (Table 5). Factors related to culture, leadership, human resources, processes and systems, and resources and facilities affect quality improvement of healthcare services.

\section{Organizational factors underpinning QI and sustainability in Saudi hospitals}

Thematic analysis revealed that participants have identified four themes and 43 sub-themes as critical success factors for quality improvement and sustainability in Saudi hospitals, as illustrated in Table 6. Success factors included: Culture, Human Resources Management, Processes and Systems, and Structure.

Table 2 The relationship between the level of QI implementation and the demographic characteristics of participants

\begin{tabular}{lllll}
\hline Variables & Characteristic & Mean \pm SD & t-test & p-value \\
\hline & Male & $16.79 \pm 4.25$ & 0.222 & 0.826 \\
& Female & $16.44 \pm 4.03$ & & 0.010 \\
Bnformation \& Analysis & Bachelor & $19.15 \pm 4.30$ & 2.709 & 0.005 \\
& Master/Doctorate & $15.67 \pm 3.69$ & & 2.98 \\
& Physician & $15.51 \pm 3.80$ & & 0.010 \\
\hline
\end{tabular}

Table 3 QI Implementation Scores

\begin{tabular}{llllllll}
\hline Variables & Sample size & Median & Mean & SD & t-test & FD & p-value \\
\hline Leadership (10) & 43 & 30 & 27.954 & 5.6986 & $-2.355-$ & 42 & 0.023 \\
Information \& Analysis (7) & 43 & 21 & 16.721 & 4.1595 & $-6.746-$ & 42 & 0.000 \\
Strategic Quality Planning (6) & 43 & 18 & 15.233 & 4.4499 & $-4.078-$ & 42 & 0.000 \\
Human Resource Utilization (8) & 43 & 24 & 20.698 & 5.4752 & $-3.955-$ & 42 & 0.000 \\
Quality Management (7) & 43 & 21 & 19.256 & 5.8395 & $-1.959-$ & 42 & 0.050 \\
Quality Results (5) & 43 & 15 & 14.163 & 3.7604 & $-1.460-$ & 42 & 0.000 \\
Customer Satisfaction (7) & 43 & 21 & 14.744 & 4.8555 & $-8.449-$ & 42 & 0.000 \\
\hline
\end{tabular}

Table 4 The relationship between Leadership and the other dimensions of Quality Improvement

\begin{tabular}{|c|c|c|c|c|c|c|c|}
\hline & LD & I\&A & SQP & HRU & QM & QR & CS \\
\hline Leadership (LD) & & {$[0.049]^{* *}$} & 0.252 & {$[0.544]^{* *}$} & {$[0.679]^{* *}$} & {$[0.469]^{* *}$} & {$[0.473]^{* *}$} \\
\hline Information \& Analysis (I\&A) & & & {$[0.718]^{* *}$} & 0.229 & 0.053 & 0.257 & 0.074 \\
\hline Strategic Quality Planning(SQP) & & & & {$[0.558]^{* *}$} & {$[0.412]^{* *}$} & {$[0.474]^{* *}$} & 0.254 \\
\hline Human Resource Utilization (HRU) & & & & & {$[0.896]^{* *}$} & {$[0.472]^{* *}$} & {$[0.712]^{* *}$} \\
\hline Quality Management (QM) & & & & & & {$[0.384]^{*}$} & {$[0.707]^{* *}$} \\
\hline Quality Results (QR) & & & & & & & {$[0.365]^{*}$} \\
\hline Customer Satisfaction (CS) & & & & & & & \\
\hline
\end{tabular}


Table 5 Factors impeding quality improvement and sustainability in Saudi hospitals

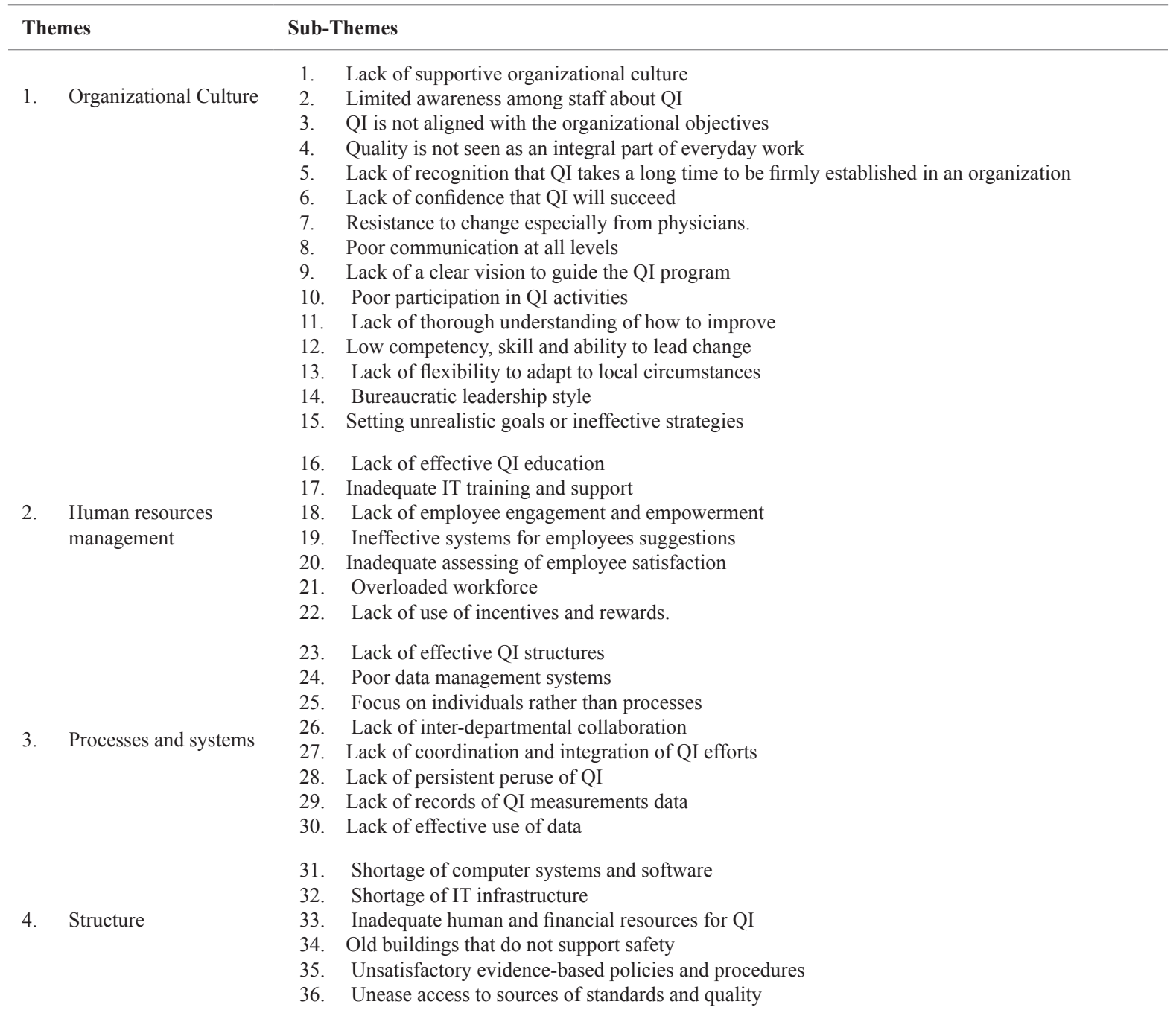

Table 6 Organizational Factors underpinning quality improvement and sustainability in Saudi hospitals

\begin{tabular}{|c|c|}
\hline Organizational Culture & $\begin{array}{l}\text { 1. Assess the organization's current culture } \\
\text { 2. Secure leadership commitment to QI } \\
\text { 3. Develop a sense of the need for change } \\
\text { 4. Establish supportive organizational structures (e.g., Quality Council) } \\
\text { 5. Assign a change team of powerful employees } \\
\text { 6. Align QI with the organizational strategic objectives } \\
\text { 7. Commit to achieving the QI goals and objectives } \\
\text { 8. Build strong belief that QI benefits staff, patients, and the organization } \\
\text { 9. Form and train Quality Improvement teams } \\
\text { 10. Focus on employees and process improvement } \\
\text { 11. Ensure safe environment that encourage staff to report errors without fear } \\
\text { 12. Ensure involvement and participation of all employees in QI activates } \\
\text { 13. Establish multi-disciplinary teams to manage and coordinate patient care } \\
\text { 14. Respect, engage and empower physicians and nurses to take lead of QI teams } \\
\text { 15. Carry out regular culture gap analysis } \\
\text { 16. Continually identify and remove barriers } \\
\text { 17. Tackle resistance to change } \\
\text { 18. Report regularly quality dashboards and scorecards to management and Board } \\
\text { 19. Make the changes show in day-to-day work and seen at all levels } \\
\text { 20. Celebrate QI successes }\end{array}$ \\
\hline
\end{tabular}


Table Continued....

\begin{tabular}{|c|c|}
\hline Themes & Sub-Themes \\
\hline $\begin{array}{l}\text { Human Resources } \\
\text { Management }\end{array}$ & $\begin{array}{l}\text { 21. Develop staff skills in data management } \\
\text { 22. Develop staff skills in data retrieval } \\
\text { 23. Emphasize QI in new employee orientation and regular staff meetings } \\
\text { 24. Establish QI training and activities as part of the daily responsibilities } \\
\text { 25. Provide opportunities for continuing education and professional advancement } \\
\text { 26. Keep vacancy rates low }\end{array}$ \\
\hline Processes and systems & $\begin{array}{l}\text { 27. Select key performance indicators } \\
\text { 28. Dedicate qualified staff to manage data } \\
\text { 29. Monitor the performance of care givers } \\
\text { 30. Compare indicators with benchmarks } \\
\text { 31. Communicate performance data } \\
\text { 32. Set the direction for the QI program } \\
\text { 33. Form multidisciplinary QI teams } \\
\text { 34. Use a systematic QI method and QI tools } \\
\text { 35. Use Plan-Do-Study-Act (PDSA) to test change } \\
\text { 36. Use QI professionals to facilitate change }\end{array}$ \\
\hline Structure & $\begin{array}{l}\text { 37. Invest in advanced medical technology } \\
\text { 38. Invest in Information Technology } \\
\text { 39. Invest in qualified QI professionals } \\
\text { 40. Develop and implement effective management plans } \\
\text { 41. Develop and implement evidence-based policies, procedures, guidelines, and protocols } \\
\text { 42. Participate in accreditation systems } \\
\text { 43. Provide ease of access to important sources for standards and best practice }\end{array}$ \\
\hline
\end{tabular}

\section{Discussion}

The challenges that face the Saudi healthcare system have been the driving force behind the study presented in this paper. Despite many years of use of various quality improvement models within the Saudi care system, healthcare services are still in need of improvement pertaining to safety, timeliness, effectiveness, and efficiency. The Saudi healthcare system involves healthcare professionals who have different backgrounds, educational qualifications, languages and cultures. The system also rests on longstanding, hierarchical and bureaucratic linear structures that are increasingly contrasted against the emerging flat, participative and transformational structures supported by the quality philosophy. One proposed solution to these challenges is quality management and improvement. However, many reports show that quality improvement in the Saudi healthcare context is far from easy. On the contrary, many efforts fail, or it is not known whether the initiatives improved quality, safety or efficiency of the care processes. Therefore, this study focuses on the organizational characteristics that impede or underpin quality improvement and sustainability in Saudi hospitals. The degree of quality improvement implementation is defined as the extent to which Saudi hospitals apply the QI concepts and practices in their management system. These practices are: leadership, information and analysis, strategic quality planning, human resource utilization, quality management, quality results, and customer satisfaction. Analyzing the current situation at the beginning of this thesis was considered necessary to identify the gap between actual and expected performance.

We examined the overall status of QI implementation in Saudi hospitals, and determined which organizational characteristics played a critical role in facilitating or preventing QI implementation. We also attempted to develop a theoretical framework to describe facilitators and inhibitors of QI implementation in Saudi hospitals. The mean score computed across the seven dimensions was $(2.52 \pm 0.50)$, which is far from the score achieved by hospitals in other countries (e.g.,
US hospitals $3.33 \pm 0.15$, Korean hospitals $3.34 \pm 0.50$ ). The mean scores for each of the seven dimensions ranged from $(2.80 \pm 0.57)$ to $(2.11 \pm 0.69)$, suggesting ample room for improvement. Of the seven dimensions, 'human resource utilization' $(2.59 \pm 0.69)$ achieved the highest score, followed by 'leadership' $(2.80 \pm 0.57)$, and 'quality management' $(2.75 \pm 0.83)$. The lowest score was registered for 'customer satisfaction' $(2.11 \pm 0.69)$, followed by 'information and analysis' $(2.39 \pm 0.59)$, and 'quality results' $(2.47 \pm 0.57)$.

Our study revealed many important findings. First of all, the QI implementation, as measured by seven critical practices, is significantly poor in Saudi hospital ( $p$-value $<0.05$ ). This means that, the quality and safety of patient care provided by Saudi hospitals fall far short of what is optimal. It also means that Saudi hospitals are facing serious challenges in implementing QI strategies and their success has been very minimal. A major finding of our study is that, based on the positive association with the other QI implementation dimensions, human resources utilization and quality management are the key drivers to successful implementation of QI in Saudi hospitals. We also found that, the QI efforts did not lead to the desired outcomes on customer satisfaction and quality results. Saudi hospitals scored very low in 'customer satisfaction' $(2.11 \pm 0.69)$ with (p-value 0.000$)$ and $(2.47 \pm 0.57)$ with (p-value 0.000$)$ in quality results. This means that, Saudi hospitals failed to meet customers' expectations or achieve sustained measurable improvements in the quality and safety of patient care. Despite the substantial resources invested by many Saudi hospitals to adapt and implement QI programs, many of them did not achieve any improvement and some only a little. This could be because Saudi hospitals utilize only a partial implementation of QI, and hence are unable to achieve continuous and systematic improvement.

We found that, strategic quality planning in Saudi hospitals is significantly inadequate scoring $(2.54 \pm 0.71)$ with (p-value 0.000$)$. This is probably because the main driver for the initiation of QI in Saudi hospitals is external pressures by the Saudi Central Board for 
Accreditation of Healthcare Institutions (CBAHI) rather than by any internal strategic business planning requirement. Our study showed that, the least implemented dimension of QI in Saudi hospitals was information and analysis with significantly low score at $(2.39 \pm$ $0.59)$ and (p-value 0.000). This explains the poor implementation of the other dimensions of QI especially strategic quality planning which is positively associated with adequate information systems and technical skills in data analysis and use. Strategic quality planning is a systematic approach to defining long-term business goals, including those to improve quality and the means to achieve them. This means that, inadequate information and analysis serves as the main barrier to strategic quality planning in Saudi hospitals which is in turn positively associated with human resources utilization, quality management and quality results. Our findings confirm previous studies about the importance of Information Technology in improving the quality and safety of patient care.

Finally, the major findings of the qualitative phase of our study confirm the presence of a multitude of organizational barriers that impede QI in Saudi hospitals. These are related to: organizational culture, human resources management, processes and systems, and structure. These four domains were found to have the strongest impact on the facilitation of QI among Saudi hospitals. They describe the organizational factors that facilitate or inhibit QI implementation and sustainability in Saudi hospitals. In particular, the level of preparation of the workforce for change, building the organizational structure for QI, linking QI goals to the organization's mission and vision, and measuring and improving performance which are the most significant organizational factors impeding QI implementation. Our findings provide empirical evidence of the best practices related to each of the four domains and how organizations can overcome the barriers to QI implementation. Any improvement in these four domains will lead to significant improvement in the implementation of all the dimensions of QI in Saudi hospitals.

Our results concur with the previous work of Albejaidi ${ }^{4}$ and Al Malki et al. ${ }^{5}$ who found that Saudi hospitals were facing serious challenges in improving the quality of services provided to citizens despite the enormous fiscal expenditure and huge resources allocated by the Saudi government to the health sector. The present research found that nothing had changed in terms of the level of quality improvement implementation in Saudi hospitals. Our findings also support the recent study of Aljuaid et al. ${ }^{3}$ who clearly highlighted the need to improve the quality of healthcare delivery in Saudi hospitals, specifically in areas of patient safety, clinical effectiveness and patient-centeredness. These findings of this study confirm previous studies by ${ }^{6-8}$ that found that the Saudi healthcare system does not provide a well-developed quality culture where quality improvement strategies and other quality initiatives can flourish. Our findings also go in line with the findings of Bayraktar et al. ${ }^{10}$ Malek \& Kanji ${ }^{11}$, $\&$ Rosa et al. ${ }^{12}$ who emphasized the need of effective and efficient employee utilization as a key tool of QI implementation in services sector, and the findings of Schalk \& Dijk ${ }^{13}$ who found that hospitals that focus on integrating quality management with human resource utilization achieve organizational excellence ${ }^{14,15}$.

\section{Limitations}

Due to the very nature of humans, even with the best of intentions in mind, the researcher is often confronted with a variety of variables which may affect the reliability of his findings. Several limitations of this study must be mentioned. Firstly, the level of QI implementation and the organizational characteristics influencing QI implementation were evaluated based on the perspective of only one or few persons responsible for QI within their hospitals and, therefore, the generalization of the results should be taken with caution. In addition, certain questions in the survey relied on the subjective judgment or perceived values of the respondent and, therefore, there is a risk that the answers do not accurately reflect the current situation. However, because the individuals chosen were considered to have expert knowledge of the situations within their organizations with respect to QI, these individuals were undoubtedly the most appropriate choice for the purpose of the survey and interviews. Moreover, the mix method used in this study improved the credibility of the results. Finally, the long experience of the researcher in eight different Saudi hospitals and his deep knowledge of the current situation in Saudi hospital helped the researcher to take the necessary measures to minimize the risk of bias. In summary, this study enabled us to assess systematically the level of QI implementation in Saudi hospitals, which are undergoing many organizational changes subsequent to the adoption of CBAHI standards. The major findings of this study confirm that the successful implementation of any QI strategy requires addressing four critical domains: organizational culture, human resources utilization, process and systems, and structure. It is hoped that this study will make a significant contribution to the body of scientific evidence on organizational influencing QI in the health care setting.

\section{Acknowledgments}

None.

\section{Conflict of interest}

The author declares that there is no conflict of interest.

\section{References}

1. Abdulrahman H Alrabeah1, Susan M Ogden, David A Edgar, et al. TQM in the Saudi Health Care System: A National Cultural Perspective. World Review of Business Research. 2015;5(2):120-136.

2. Al Masah Capital Management Limited, 2011. MENA: Healthcare Sector Report; UAE. 2011.

3. Aljuaid M, Mannan F, Chaudhry Z, et al. Quality of care in university hospitals in Saudi Arabia: a systematic review. BMJ Open. 2016;6(2):e008988.

4. Albejaidi FM. Healthcare System in Saudi Arabia: An Analysis of Structure, Total Quality Management and Future Challenges. Journal of Alternative Perspectives in the Social Science. 2010;2:(2):794-818.

5. Almalki M, Fitzgerald G, Clark M. Health care system in Saudi Arabia: an overview. Eastern Mediterranean Health Journal. 2011;17:(10)

6. Al-Ahmadi H, Roland M. Quality of primary health care in Saudi Arabia: a comprehensive review. International Journal for Quality in Health Care. 2005;17(4):331-346.

7. Jannadi B, Al-Shammari H, Khan A, et al. Current Structures and Future Challenges for the Healthcare System in Saudi Arabia. Asia Pacific Journal of Health Management. 2008;3(1):43-50.

8. Walston S, Al-Harbi Y, Al-Omar B. The Changing Face of Healthcare in Saudi Arabia. Ann Saudi Med. 2008;28(4):243-250.

9. Quinn RE, JR Kimberly. Paradox, Planning, and Perseverance: Guidelines for Managerial Practice. In: JR Kimberly, RE Quinn, editors. Homewood IL: Dow Jones-Irwin;1984:295-313. 
10. Bayraktar E, Tatoglu E, Zaim S. An instrument for measuring the critical factors of TQM in Turkish higher education. Total Quality Management. 2008;19:(6):551-574

11. Malek A, Kanji G. TQM in Malaysian Higher Education Institutions. Sinergie rapporti di ricerca. 2000;9(4-5).

12. Rosa MJ, Amaral A. A Self-Assessment of Higher Education Institutions from the Perspective of the EFQM Excellence Model. Quality Assurance in Higher Education. 2007;20(3):181-207.
13. Schalk R, Dijk WV. Quality management and employee commitment illustrated with examples from Dutch health care. Int J Health Care Qual Assur Inc Leadersh Health Serv. 2005;18(3):170-178.

14. Altuwaijri MM. Electronic-health in Saudi Arabia. Just around the corner?. Saudi Med J. 2008;29(2):171-178.

15. Saudi Central Board for Accreditation of Healthcare Institutions. National Standards for Hospitals, 2016 